\title{
Journal of Atomic and Molecular Sciences
}

ISSN 2075-1303

\section{Enhancement in above-threshold ionization spectrum}

Received: May 3, 2017, Accepted: June 16, 2017,

DOI: $10.4208 /$ jams.050317.061617a

http://www.global-sci.org/jams/

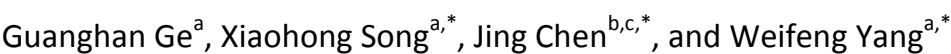

Abstract. Freeman resonance and resonance-like enhancement (RLE) in above-threshold ionization (ATI) spectrum are reviewed in this work. Two different interpretations accounting for the high-energy enhancements in the ATI spectra are described. One of them is the multiphoton ionization "channel closing" relating to the destructive/constructive interference, while the other pursue the fundmental concept of resonance and associates the enhancement with a resonant excited state. Primary calcualtion of the RLE using generalized quantum trajectory Monte Carlo method is also present.

\section{Introduction}

Above-threshold ionization (ATI), which was firstly observed in 1979 [1], has been identified as the core of strong-field physics and the most important process in the interaction between atoms and intense laser field. ATI is the process that an atom in an intense laser field absorbs $s$ more photons than the minimum number $n$ for reaching to the ionization threshold $I_{0}$. The ionization peaks separated by the energy of one photon are present in ATI spectra. The energies of the ATI peaks are given by

$$
E=(n+s) \omega-I_{0}
$$

Freeman resonance was found in experiment thirty years ago [2], and sub-peaks associated with certain excited states being resonant with the ground state are shown in the lowenergy part of the ATI spectra. If the photoelectron does not interact with the parent ion after it is born in the field, the gaining energy of it cannot exceed $2 U_{P}\left(U_{P}=l / 4 \omega^{2}\right.$ denotes the ponderomotive energy, where $I$ is the laser intensity and $\omega$ is the angular frequency). In the energy region higher than $2 U_{P}$, the rescattered photoelectrons are dominated and the electron spectrum shows a plateau. After the observation of Freeman resonance, the magnitudes of peaks in the highenergy ATI spectra around $5 \sim 10 U_{P}$ were found to exhibit strong variations when the laser intensity changes slightly [3][4]. Since the low-energy enhancement mechanism is reminiscent of the excited state resonances, the enhancement in high-energy region is attributed to the Rydberg resonances. Numerical solution of time-dependent Schrödinger equation (TDSE) well reproducing the ATI spectra [5][6][7], confirmed that the rescattering of electron wave packet from excited state leads to the enhancement feature [5].

However, opposing strong-field approximation (SFA) calculations with a zero-range potential, for which no excited state exist, can also reproduce the enhancements in the plateau [8][9][10]. This is interpreted in terms of the constructive interference of quantum trajectories when $n$ -

\footnotetext{
a. Department of Physics, College of Science, Shantou University, Shantou, Guangdong 515063, China.

b. HEDPS, Center for Applied Physics and Technology, Peking University, Beijing 100084, China.

Institute of Applied Physics and Computational Mathematics, P. O. Box 8009, Beijing 100088, China.

"Corresponding author, songxh@stu.edu.cn

Corresponding author, chen_jing@iapcm.ac.cn

Corresponding author, wfyang@stu.edu.cn
}

photon ionization channel is closing. To contribute to the debate of the two contradicting interpretations, intensitydependent ATI spectra in the direction of the polarization and those in the direction perpendicular to the polarization are compared, supporting the latter [11]. On the other hand, TDSE calculations with a long-range potential confirm that the enhancement in the high-energy part of ATI spectra originates from multiphoton resonance between the ground state and Rydberg states, and laser-induced states were proposed instead of ruling out the validity of channel closing [12]. Very recently, experiments on strong-field excitation together with TDSE calculations try to combine the seemingly opposing pictures, i.e., channel closing and rescattered electron's resonances with Rydberg states [13].

While the enhancement in the high-energy ATI spectra of rare-gas atoms do not have a unified theoretical interpretation yet, experimental observations of such enhancements were reported in simple molecules [14][15]. Recently, the enhancement of polyatomic molecules was observed [16][17]. Theoretical calculations using the SFA reproduced the experimental results and attributed the different characteristics of different molecules to interference effects of molecular orbitals with different symmetries [16], which, in fact, supported the channel closing mechanism.

In this paper, we will describe the enhancement both in low-energy and high-energy parts of the ATI spectra. The two distinct interpretations accounting for enhancement of the ATI peaks in the high-energy part of ATI spectra will be reviewed. Recent progress and applications in resonance structures will be introduced.

\section{Freeman resonance}

In this section, we first look back on the Freeman resonance [2], and then introduce the observation of Freeman resonance delay between the photoelectrons emitted via the fielddressed Rydberg states [18].

\subsection{Freeman resonance}

Figure 1 shows the first measurements of Freeman resonance [2]. When the pulse duration is less than 1 picosecond (ps), the peaks in low-energy part of ATI spectra break up into a series of narrow peaks. For long pulses, the ponderomotive energy of the photoelectron when it exits form the interaction volume counteracts the decreased kinetic energy due to the raised ionization potential. Therefore, it is impossible to find the shift 
effect of ionization potential with long pulse. When the pulse duration is short enough, there is no time for the escaped photoelectron to accelerate and the spectrum faithfully records the photoelectron energies and momentum distributions at the moment of ionization. Excited states of atom shift upward along with the ionization potential by $U_{p}$. The energy shift of an intermediate bound state is

$$
E=(n+s) \omega-I_{0}-\left(m \omega-E_{i}\right) \text {, }
$$

where $E_{i}$ is the original energy of the state and $m$ is the number of photons resonant with the state. The fine structure and the corresponding ATI peaks of Freeman resonance are shown in Fig. 2 [2].

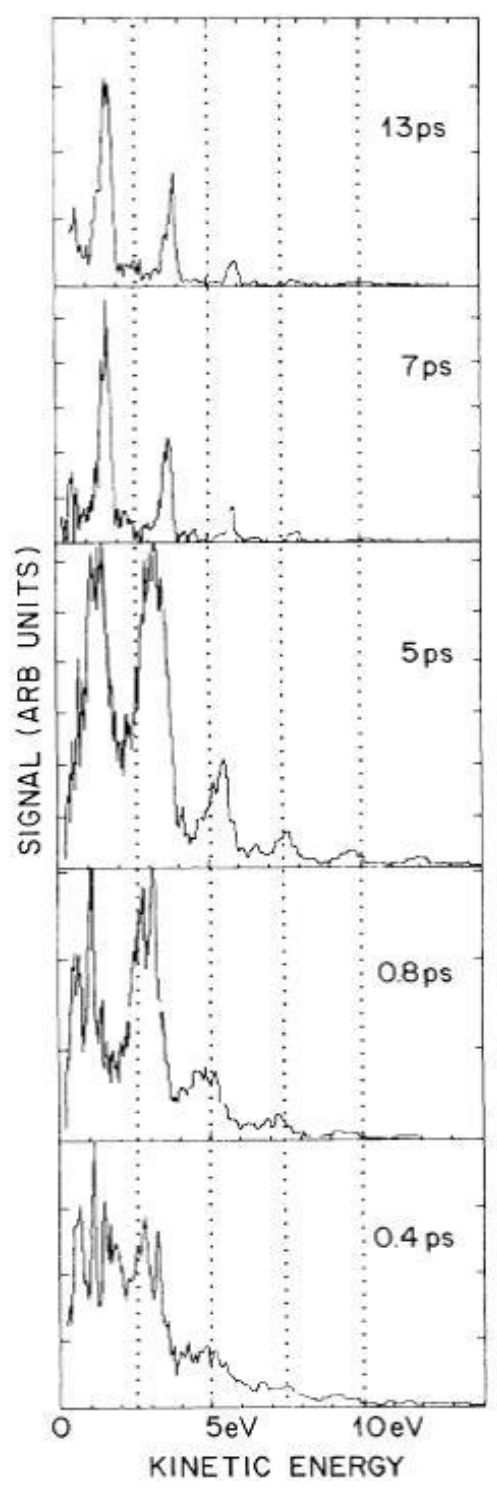

Figure 1: Kinetic energy of photoelectrons emitted from xenon as a function of pulse width. The intensity increases from $1.2 \times 10^{13} \mathrm{~W} / \mathrm{cm}^{2}$ for pulse width $15 \mathrm{ps}$ to $3.9 \times 10^{14} \mathrm{~W} / \mathrm{cm}^{2}$ for $0.4 \mathrm{ps}$ (adapted from Ref. [2])

\subsection{Attosecond time delay of Freeman resonance}

In this subsection, we will introduce the recent measurements of attosecond time delay of Freeman resonance. A phasecontrolled orthogonal two-color (OTC, 800-400nm) femtosecond laser pulse was employed to probe the photoelectron emission delay in multiphoton ATI of an atom [18]. Figure 3 shows the measured, the generalized quantum trajectory Monte Carlo (GQTMC) [20] and the TDSE $\phi_{L^{-}}$ integrated ( $\phi_{L}$ is the relative phase of the OTC pulse) photoelectron angular distributions of singly ionized argon (Ar). Along $y$-axis $\left(\phi_{\mathrm{e}}=0^{\circ}\right.$ or $\pm 180^{\circ}, \phi_{\mathrm{e}}$ is the emission angle of the electron) only main ATI peaks (labeled by white dots) spaced by $400 \mathrm{~nm}$ photon energy is observed. Additional sidebands differed by a photon energy of the $800 \mathrm{~nm}$ field between two adjacent main ATI peaks appear for electrons emitted away from the y-axis, e.g. $\phi_{\mathrm{e}}=30^{\circ}$ (labeled by black dots).

The GQTMC simulations, which have well reproduced different experimental and TDSE results with different parameters [21][22][23], agreed with the experimental observations here (see Fig. 3(a) and (b)). The Freeman resonance is not able to be included in the GQTMC simulations without considering excited states. While other processes, such as the time delay induced by the multiphoton transition process and the free electron propagation in the combined field of the atomic potential and the laser field, can be described accurately. To estimate the Freeman resonance delay we ran the TDSE simulations [24][25][26] (see Fig. 3(c)) in parallel to the GQTMC simulations. The Freeman resonance delay is revealed by subtracting the phase difference between the experimental measurements (or TDSE) and GQTMC simulations. We observed a $(0.19 \pi+0.22 \pi) / 2=0.21 \pi \approx 140$ attoseconds difference of time delay of photoelectrons emitted via the field-dressed Freeman resonant $4 f$ and $5 p$ Rydberg states of Ar. We summarize the experimentally measured and simulated phase differences between two photoionization pathways in Table 1 . The data in the two rows corresponds to electrons emitting to $\phi_{\mathrm{e}}=0^{\circ}$ and $\phi_{\mathrm{e}}=30^{\circ}$.

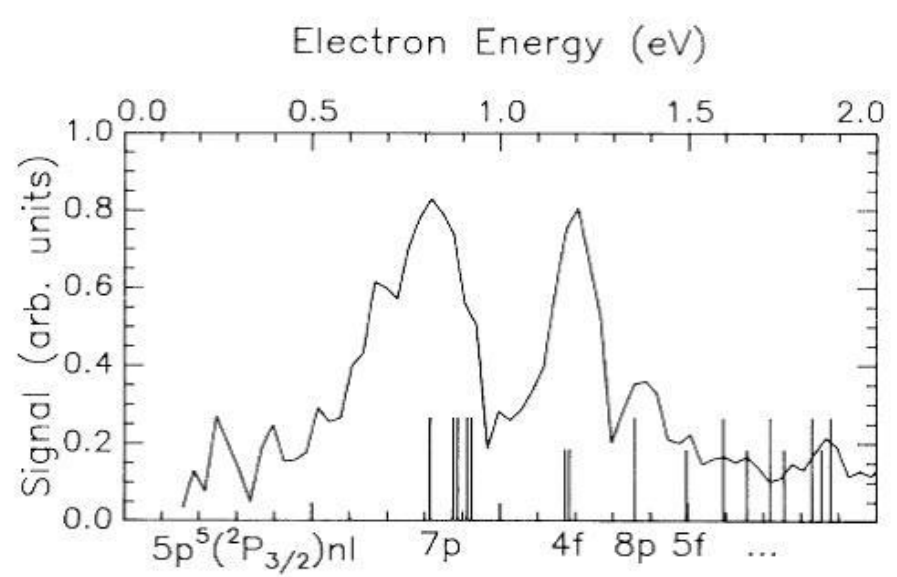

Figure 2: $s=0$ fine-structure date for pulse width less than $0.5 \mathrm{psec}$ : also plotted is the location, according to Eq. (3), of the relevant $5 p^{5}\left({ }^{2} p_{3 / 2}\right) n /$ states from Moore (Ref. [19]). The instrument resolution, which increases as the square root of the energy, is $0.05 \mathrm{eV}$ at $0.5 \mathrm{eV}$ and $0.1 \mathrm{eV}$ at $2.0 \mathrm{eV}$. With the exception of the missing peak corresponding to the $5 p^{5}\left({ }^{2} p_{3 / 2}\right) 10 p(1.72 \mathrm{eV})$ state, the experimental spectrum is reasonably well assigned above $0.8 \mathrm{eV}$. As discussed in the text, the peak in the spectrum below $0.8 \mathrm{eV}$ may have their origin in the blend of a large number of states, including $m=5$ odd-parity resonances (adapted from Ref. [2]).

Table 1: Experimentally measured and simulated phase differences between two photoionization pathways, i.e. photoelectrons emitted via the $4 f$ and $5 p$ states of $\mathrm{Ar}$ (adapted from Ref. [18]).

\begin{tabular}{|c|c|c|c|c|c|}
\hline \multirow[b]{2}{*}{$\begin{array}{c}\text { Emission } \\
\text { angle } \\
\phi_{e}\end{array}$} & \multirow[b]{2}{*}{$\begin{array}{c}\text { Experiment } \\
\Delta \phi_{p p}^{\exp }= \\
\left|\phi_{p p}^{4 f}-\phi_{p p}^{5 p}\right|\end{array}$} & \multirow[b]{2}{*}{$\begin{array}{c}\text { TDSE } \\
\Delta \phi_{P P}^{\text {TDSE }}= \\
\left|\phi_{P P}^{22 e V}-\phi_{P P}^{18 e v}\right|\end{array}$} & \multirow[b]{2}{*}{ 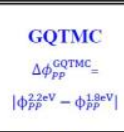 } & \multicolumn{2}{|c|}{ Freeman resonance delay difference } \\
\hline & & & & $\begin{array}{c}\text { Exp.-GQTMC } \\
\Delta \phi_{p p}^{4 f / 5 p}= \\
\left|\Delta \phi_{p p}^{\exp }-\Delta \phi_{p p}^{\mathrm{CQTMC}}\right|\end{array}$ & $\begin{array}{c}\text { TDSE-GQTMC } \\
\Delta \phi_{p p}^{4 / 5 p}= \\
\left|\Delta \phi_{p P}^{\mathrm{TPSE}}-\Delta \phi_{p P}^{\mathrm{CQTMC}}\right|\end{array}$ \\
\hline $0^{\circ}$ & $0.24 \pi$ & $0.19 \pi$ & $0.05 \pi$ & $0.19 \pi$ & $0.14 \pi$ \\
\hline $30^{\circ}$ & $0.42 \pi$ & $0.33 \pi$ & $0.20 \pi$ & $0.22 \pi$ & $0.13 \pi$ \\
\hline
\end{tabular}




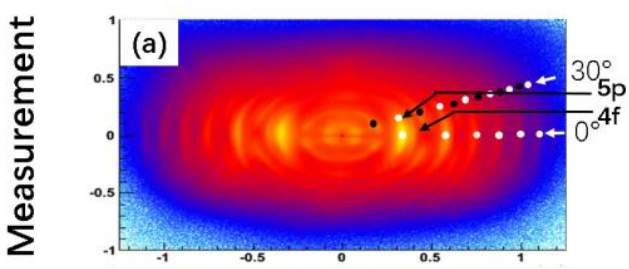

(b)
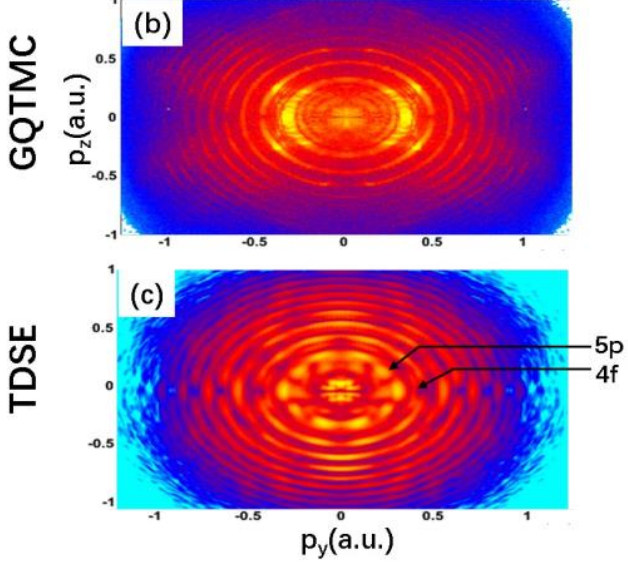

Figure 3: (a) Measured (b) GQTMC, and (c) TDSE $\phi_{L}$-integrated ( $\phi_{L}$ is the relative phase of the OTC pulse) photoelectron angular distributions of singly ionized $\mathrm{Ar}$ (adapted from Ref. [18]).

\section{Resonance-like enhancements}

\subsection{Analysis based on resonance}

In addition to Freeman resonance in low-energy part of ATI spectra, conspicuous enhancement of ionization peaks in the high-energy part of ATI spectra was found [3][4]. Figure 4 shows the ATI spectrum for $A r$ at a laser intensity of $7 \times 10^{13} \mathrm{~W} / \mathrm{cm}^{2}$. The low-energy part of the ATI spectrum is dominated by enhancements of Freeman resonance which is 11-photon resonance via the field-dressed $g$ state of Ar. The high-energy region around $15-30 \mathrm{eV}$ shows a large secondary maximum in the ATI envelope (see the right-hand inset of Figure 4). The subsequent TDSE simulation for a model Ar atom faithfully reproduced the ATI spectrum and the enhancement feature in high-energy region [5][6][7].

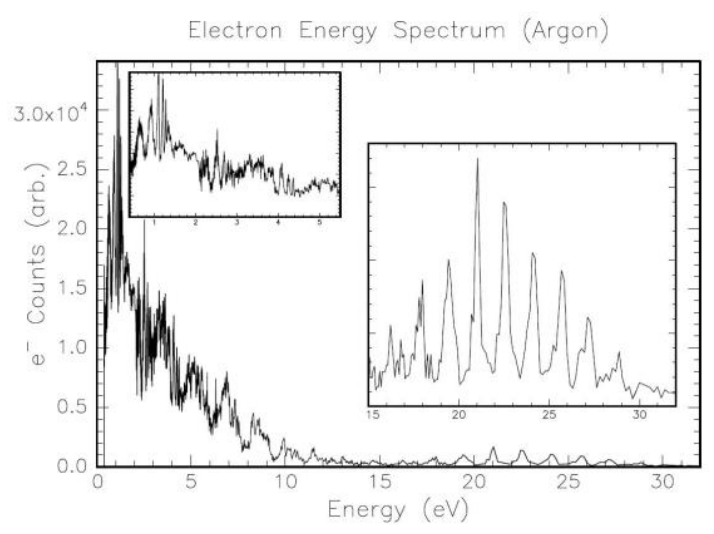

Figure 4: ATI spectrum for argon at an intensity of about $7 \times 10^{13} \mathrm{~W} / \mathrm{cm}^{2}$. The left-hand inset shows the region of low-order Rydberg resonances with an expanded energy scale. The right-hand inset shows the region of high-energy electron which are the focus of this paper (adapted from Ref. [3])
To clarify the nature of the observed enhancement, the ground state was removed from the whole wave function. Figure 5 shows the electron density of the residual wave function 0.15 optical cycle after a zero crossing of the electric field. The wave function evolves into several probability bunches most of which escape to infinity. In the resonance case, the two innermost bunches (labelled by star mark) with almost zero drift velocity, are driven back towards the parent ion and collide with it to produce a burst of high-energy electrons on backscattering. The population of the two inner bunches is strongly enhanced at resonance (Figure 5(a)), and is suppressed without resonance (Figure 5(b)) [5].

\subsection{Channel closing}

The above analysis based on TDSE simulation seems to confirm the picture of excited state resonance. However, the enhancement feature in high-energy spectra has also been reproduced by SFA calculations with a zero-range potential [8][9][10][27][28][29]. It should be noted that only one bound state, i.e. the ground state, is included and no resonance is involved in this kind of SFA calculations. The energy of the ATI electron spectrum is

$$
E=n \omega-U_{P}-I_{0} \text {. }
$$

Here, the Stark shift of the ground-state have been neglected. According to the equation (3), more photons are required with increasing laser intensity. If the condition $U_{P}+I_{0}=k \omega$ is satisfied the $k$-photon ionization is no longer sufficient and $k+1$ photons are needed, which is the $k$-photon channel closing [10].

Figure 6 shows the calculated ATI spectrum with SFA and explores the effects of the channel closings. $k=36$ (lower panel), 37 (middle panel) and 38 (upper panel) channel closings are investigated in this figure. For the two even-order channel closings, there are two enhancements: one of them between 100 and $200 \mathrm{eV}$ are very pronounced, and the other less well developed one locates between 260 and $290 \mathrm{eV}$. In the oddorder case, there is only one enhancement between 100 and $160 \mathrm{eV}$.

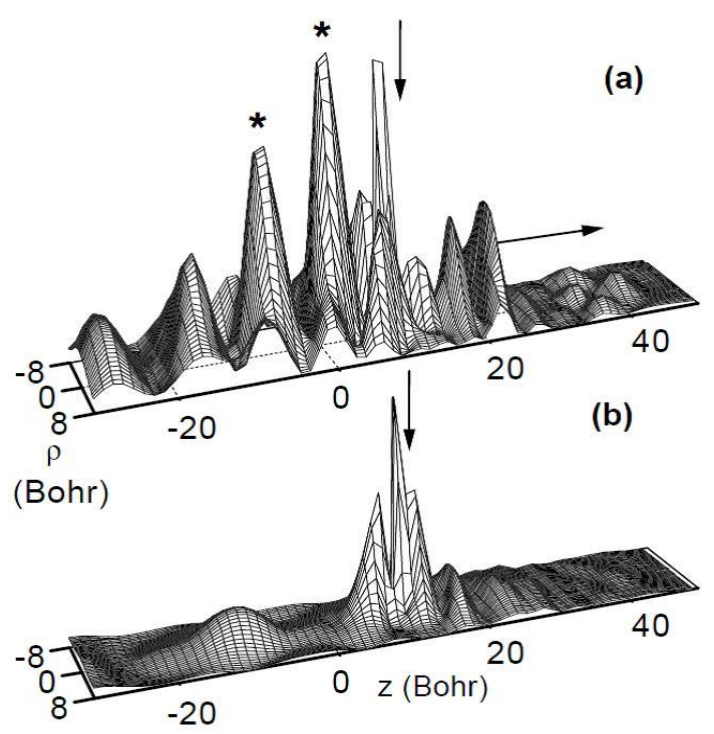

Figure 5: Electron density left after removal of the ground state at (a) $E_{0}=$ 0.0440 (on resonance) and (b) $E_{0}=0.0425$ (off resonance). The main difference between the two cases is the presence of two lobes (marked ${ }^{*}$ ) with near-zero drift velocity, from which fast electrons (visible at about $z=20$ ) have been scattered as the lobes encountered the nucleus (vertical arrows) (adapted from Ref. [5]). 


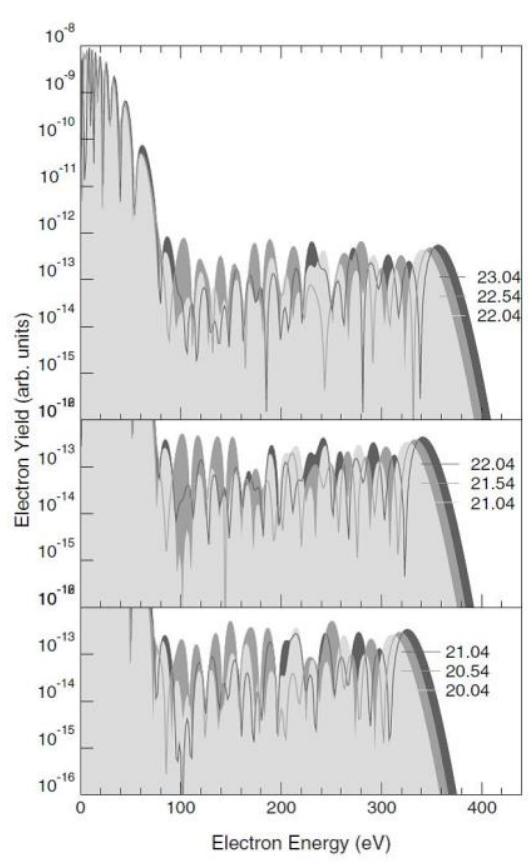

Figure 6: Envelopes of ATI spectra in the direction of the laser field for $E_{0}=-0.881$ au (corresponding to helium) and $\omega=0.057 \mathrm{au}$. Three channel closings are exlored: $\mathrm{k}=36$ (lower panel), 37 (middle panel), and 38 (upper panel). Each panel presents the spectrum for the channel-closing intensity (shaded in medium gray), and for one intensity below the former (light gray), and one above (dark gray). The intensities are identified by the corresponding values of $\eta: \eta=U_{P} / \omega=21.54$ corresponds to $5.6 \times 10^{14}$ $\mathrm{W} / \mathrm{cm}^{2}$. This figure should be compared to figure 1 of [9] (adapted from Ref [10]).

\section{GQTMC calculated ATI spectra}

Based on the previous investigations, we have enough reasons to believe that the resonance enhancement is related to the rescattering and the interference of electron wave packets integrated into the GQTMC method. The calculated ATI spectra along the direction of laser polarization are present in Figure 7. It is found that the resonance-like enhancement can also be reproduced by the GQTMC simulation. The enhancement appears between 25 and $30 \mathrm{eV}$ and the maximal peak locates around $28 \mathrm{eV}$ in the ATI spectrum at intensity of $7 \times 10^{13} \mathrm{~W} / \mathrm{cm}^{2}$. For the other two intensities, i. e., $6.5 \times 10^{13} \mathrm{~W} / \mathrm{cm}^{2}$ and $7.5 \times 10^{13} \mathrm{~W} / \mathrm{cm}^{2}$, the maximal peaks are apparently less pronounced and locate around $27 \mathrm{eV}$ and $29 \mathrm{eV}$, respectively (see inset). It is noteworthy that the ionic Coulomb potential, ignored in the SFA, is taken into account in the GQTMC calculation.

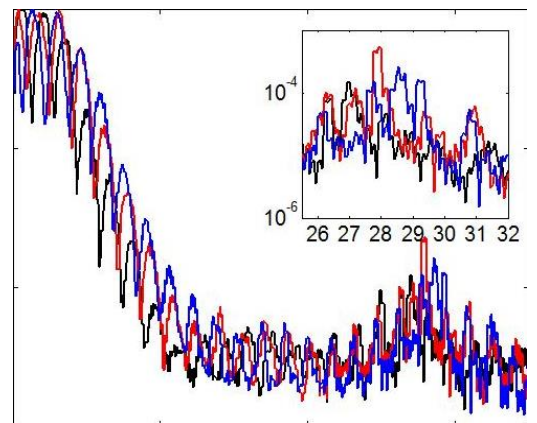

Figure 7: Computed ATI spectra of $\mathrm{Ar}$ in the direction the laser field for intensities $6.5 \times 10^{13} \mathrm{~W} / \mathrm{cm}^{2}$ (black line), $7.0 \times 10^{13} \mathrm{~W} / \mathrm{cm}^{2}$ (red line) and $7.5 \times$ $10^{13} \mathrm{~W} / \mathrm{cm}^{2}$ (blue line). The inset shows the enlarged regime of resonance-like enhancements.

\section{Concluding remarks}

Since the enhancement was firstly observed three decades ago [2], through experimental and theoretical efforts our understanding of this feature in ATI spectra has greatly advanced. However, two distinct interpretations accounting for enhancement in the high-energy part of ATI spectra, i.e. the multiphoton resonance involving excited states, and channel closings, were proposed. The underlying physics of the enhancement has been the source of debate but no consensus has been achieved so far [12][13][17]. A more rigorous theoretical analysis with consideration of the atomic potential effect and recollision is strongly required, and the GQTMC method may be a good candidate to further explore the underlying physics.

\section{Acknowledgements}

The work was supported by the National Key Program for S\&T Research and Development (Grant No. 2016YFA0401100), the National Basic Research Program of China (Grant No. 2013CB922201), the NNSF of China (Grant No. 11374202, No. 11674209 , No. 11274220 , No. 11274050 , No. 11334009 and 11425414), the Major Program of Guangdong Natural Science Foundation (Grant No. 2014A030311019), and the Open Fund of the State Key Laboratory of High Field Laser Physics (SIOM). W. Y. acknowledges support by the "YangFan" Talent Project of Guangdong Province.

\section{References}

[1] P. Agostini, F. Fabre, G. Mainfray, and G. Petite, Phys. Rev. Lett. 42 (1979) 1127.

[2] R. R. Freeman, P. H. Bucksbaum, H. Milchberg, S. Darack, D. Schumacher, and M. E. Geusic, Phys. Rev. Lett. 59 (1987) 1092

[3] Marcus P Hertlein, Philip H Bucksbaum and H G Muller, J. Phys. B. 30 (1997) L197.

[4] P. Hansch, M. A. Walker, and L. D. Van Woerkom Phys. Rev. A 55 (1997) R2535.

[5] H.G. Muller and F.C. Kooiman, Phys. Rev. Lett. 81 (1998) 1207.

[6] M. J. Nandor, M. A. Walker, and L. D. Van Woerkom, Phys. Rev. A 60 (1999) R1771

[7] H. G. Muller, Phys. Rev. A 60 (1999) 1341

[8] R. Kopold and W. Becker, J. Phys. B. 32 (1999) L419.

[9] G. G. Paulus, F. Grasbon, A. Dreischuh, and H. Walther, Phys. Rev. Lett. 84 (2000) 3791.

[10] R. Kopold, W. Becker, M. Kleber and G. G. Paulus, J. Phys B. 35 (2002) 217.

[11] E. Cormier, D. Garzella, P. Breger, P. Agostini, G. Chériaux and C. Leblanc, J. Phys. B. 34 (2001) L9.

[12] Joseph Wassaf, Valerie Veniard, Richard Taieb, and Alfred Maquet, Phys. Rev. Lett. 90 (2003) 013003.

[13] H. Zimmermann, S. Patchkovskii, M. Ivanov and U. Eichmann, Phys. Rev. Lett. 118 (2017) 013003.

[14] C. Cornaggia, Phys. Rev. A 82 (2010) 053410.

[15] W. Quan, X. Y. Lai, Y. J. Chen, C. L. Wang, Z. L. Hu, X. J. Liu, X. L. Hao and J. Chen, Phys. Rev. A 88 (2013) 021401(R)

[16] C. Wang, Y. Tian, S. Luo, W. G. Roeterdink, Y. Yang and D. Ding, Phys. Rev. A 90 (2014) 023405. 
[17] C. Wang, M. Okunishi, X. Hao, Y. Ito, J. Chen, Y. Yang, R. R. Lucchese, M. Zhang, B. Yan, W. D. Li, D. Ding, and K. Ueda, Phys. Rev. A 93 (2016) 043422.

[18] X. C. Gong, C. Lin, F. He, Q. Y. Song, K. Lin, Q. Y. Ji, W. B. Zhang, J. Y. Ma, P. F. Lu, Y. Q. Liu, H. P. Zeng, W. F. Yang and J. Wu, Phys. Rev. Lett. 118 (2017) 143203.

[19] C. E. Moore, Atomic Energy Levels, U. S. National Bureau of Standards Circular No. 467 (U. S. GPO, Washington, DC,1949)

[20] X. H. Song, C. Lin, Z. H. Sheng, P. Liu, Z. J. Chen, W. F. Yang, S. L. Hu, C. D. Lin, J. Chen, Sci. Rep. 6 (2016) 28392.

[21] X. H. Song, J. W. Xu, C. Lin, Z. H. Sheng, P. Liu, X. H. Yu, H. T. Zhang and W. F. Yang et al, Phys. Rev. A 95 (2017) 033426.

[22] W. Yang, H. Zhang, C. Lin, J. Xu, Z. Sheng, X. Song, S. Hu and J. Chen, Phys. Rev. A 94 (2016) 043419.

[23] C. Lin, H. Zhang, Z. Sheng, X. H Yu, P. Liu, J. Xu, X. Song, S. Hu, J. Chen, W. Yang, Acta Phys. Sin 65 (2016) 223207.

[24] W. Yang, Z. Sheng, X. Feng, M. Wu, Z. Chen and X. Song, Opt. Express 22 (2014) 2519.

[25] W. Yang, X. Song and Z. Chen, Opt. Express 20 (2012) 12067.

[26] W. Yang, X. Song, Z. Zeng, R. Li and Z. Xu Opt. Express 18 (2010) 2558.

[27] G. G. Paulus, F. Grasbon and H. Walther, Phys. Rev. A 64 (2001) 021401(R).

[28] Bogdan Borca, M. V. Frolov, N. L. Manakov and Anthony F. Starace, Phys. Rev. Lett. 88 (2002) 193001.

[29] S. V. Popruzhenko, Ph. A. Korneev and S. P. Goreslavski, Phys. Rev. Lett. 89 (2002) 023001. 